\title{
TR4 Inhibits LXR-mediated Decrease of Lipid Accumulation in 3T3-L1 Adipocytes
}

\author{
Hojung Choi and Eungseok Kim* \\ Department of Biological Sciences, Chonnam National University, \\ Gwangju 500-757, Korea
}

\begin{abstract}
TR4 has been suggested to play an important role in lipid metabolism in adipocytes. Although TR4 facilitates lipid accumulation during adipogenesis, the regulatory effect of TR4 on lipid storage in mature adipocytes remains unclear. We showed that TR4 inhibited the LXR agonist GW3965-mediated decrease of lipid accumulation in 3T3-L1 adipocytes. A reporter gene analysis revealed that TR4 suppressed LXR $\alpha$ transcriptional activity, although LXR $\alpha$ was unable to affect TR4 transcriptional activity. Moreover, adding TR4 resulted in reduced LXR $\alpha$ binding to the LXR responsive element in a gel shift assay. Additionally, the suppressive effect of GW3965 on perilipin expression and lipid accumulation in 3T3-L1 adipocytes was abolished by TR4 overexpression. Taken together, our data demonstrate that TR4 plays an inhibitory role in LXR $\alpha$-mediated suppression of lipid accumulation in 3T3-L1 adipocytes. This TR4 protective effect is mediated, in part, by blocking the suppressive effect of GW3965 on perilipin gene expression.
\end{abstract}

Key words: Testicular orphan nuclear receptor 4, liver X receptor, adipocyte, perilipin, and lipid accumulation

\section{Introduction}

Adipocytes are the cells critical for energy homeostasis. Adipocytes store the extra lipids as fat during energy excess and mobilize them at the period of the energy deprivation. Dysregualtion of lipid homeostasis in adipocytes by altered expression of genes involved in lipid metabolism is a major risk factor for the obesity and diabetes, hypertension, and coronary artery disease (Reaven et al., 1988). Epidemiological studies indicate that diets containing high proportion of saturated fatty acids, are also considered as a risk factor for development and progression of these diseases since high levels of saturated fatty acids cause insulin resistance and consequently increase the risk of these diseases (Hunnicutt et al., 1994). Thus, fatty acid profile in dietary fat including milk fat is also considered important.

Testicular orphan nuclear receptor 4 (TR4; Nr2c2), a member of the nuclear receptor superfamily, is able to regulate the expression of target genes through binding to

\footnotetext{
*Corresponding author: Eungseok Kim, Department of Biological Sciences, Chonnam National University, Gwangju 500757, Korea. Tel: 82-62-530-3402, Fax: 82-62-530-3409, Email: ekim@chonnam.ac.kr
}

direct repeats (DRs) of AGGTCA core motifs with variable numbers of spacer nucleotides (Kim et al., 2003; Lee et al., 1998a; Lee et al., 1995; Lee et al., 1997; Lee et al., 1999). Several studies suggested that TR4 may play key roles in glucose and lipid metabolism (Liu et al., 2007). Recently, we found that knockdown of TR4 in 3T3-L1 adipocytes resulted in decrease of intracellular lipid accumulation via downregulation of fatty acid transport protein 1 and perilipin genes. In addition, we also reported that TR4-deficient mice showed reduced fat mass and lipid deposition in the liver with decreased expression of hepatic stearoyl-CoA desaturase 1 (SCD1) (Kim et al., 2011a). SCD1 is the rate-limiting enzyme in the biosynthesis of monounsaturated fatty acids (Kim et al., 2011b). SCD1 transgene in goats alters milk fatty acid composition to less saturated fatty acid content (Zidi et al., 2010).

The liver X receptor (LXR) is initially identified in the liver and has a critical role in foam cell formation through regulation of genes involved in reverse cholesterol transport (Ory, 2004; Willy et al., 1995). In the liver, LXR facilitates conversion of cholesterol to bile acids by induction of cholesterol $7 \alpha$-hydroxylase (Ory, 2004). LXR also regulates genes required for lipid metabolism such as sterol-regulatory element binding protein $1 \mathrm{c}$, fatty acid synthase, lipoprotein lipase, and SCD1. Given the 
established role of LXR in lipid metabolism in the liver, LXR is considered to play a role in adipogenesis. However, conflicting results about the role of LXR in adipogenesis have been reported. Several studies have reported that LXR is significantly induced in the early phase of differentiation and participates in adipogenesis (Seo et al., 2004; Ulven et al., 2005). In contrast, a recent study shows that LXR decreases fat storage in the mature adipocytes in part by increase of basal lipolysis (Stenson et al., 2011). LXR forms a heterodimer with retinoid $\mathrm{X}$ receptor (RXR) and regulates expression of target genes via direct binding to DR4 response elements located in the target gene promoters (Apfel et al., 1994). Recently, LXR and thyroid receptor have shown to regulate carbohydrate responsive element-binding protein expression through competition for DR4 located in the promoter of carbohydrate responsive element-binding protein gene (Hashimoto et al., 2009). TR4 also participates in other nuclear receptor signaling pathways through DNA binding competitions to their target genes or protein-protein interaction (Lee et al., 1998b; Shyr et al., 2002), strongly suggesting that TR4 and other nuclear receptors such as LXR may construct the regulatory network for lipid homeostasis in adipocytes. Since both TR4 and LXR activate SCD1 expression and have binding affinity for DR4 response elements, it will be interesting to study the possible cross-talk between TR4 and LXR to understand the regulatory network for lipid homeostasis in adipocytes. Here, we demonstrate that TR4 suppresses LXR $\alpha$-mediated inhibition of lipid accumulation in 3T3-L1 adipocytes through competitive regulation of perilipin gene expression. Taken together, cross-talk between TR4 and LXR forms a regulatory network to maintain lipid homeostasis which is important for adipocyte biology.

\section{Materials and Methods}

\section{Cell cultures and adipocyte differentiation}

Cells were maintained in DMEM medium supplemented with $10 \%$ fetal bovine serum (HEK293T) or 10\% newborn calf serum (3T3-L1 and NIH-3T3). 3T3-L1 cells stably transfected with pcDNA3, pcDNA3-TR4, pcDNA $^{\mathrm{TM}} 6.2-\mathrm{GW} / \mathrm{miR}$, or pcDNA ${ }^{\mathrm{TM}} 6.2-\mathrm{GW} / \mathrm{TR} 4 \mathrm{miR}$ were maintained in DMEM containing $800 \mu \mathrm{g} / \mathrm{mL}$ Geneticin or $10 \mu \mathrm{g} / \mathrm{mL}$ Blastacidin. Two days after confluence (designated day 0), 3T3-L1 cells were differentiated using $10 \%$ FBS DMEM containing $5 \mu \mathrm{g} / \mathrm{mL}$ insulin, $0.25 \mathrm{mM}$ 3-isobutyl-1-methylxanthine (IBMX), and $0.25 \mu \mathrm{M}$ dexamethasone. Seventy-two hour later, the medium was replaced by adipocyte medium containing $5 \mu \mathrm{g} / \mathrm{mL}$ insulin and $0.25 \mu \mathrm{M}$ dexamethasone. Oil Red $\mathrm{O}$ staining was performed as previously described (Kim et al., 2010).

\section{Plasmids and reporter gene assay}

Plasmids pCMX-TR4, pCMX-LXR $\alpha$, pCMX-RXR $\alpha$, pCMX-VP16-RXR $\alpha$, pCMV-VP16-TR4, pG5-Luc, TR4RELuc, and LXRE-Luc were described previously (Kim et al., 2003; Willy et al., 1995). pCMX-Gal4-LXR $\alpha$ was constructed by cloning of ligand binding domain amplified by PCR from pCMX-LXR $\alpha$. Transfections were performed by using SuperFect (Qiagen). Relative luciferase activity (fold) was expressed based on the induction relative to the transfection of empty vector (set as 1-fold) without agonist; the results are mean \pm standard deviation (SD) of three separate experiments.

\section{Reverse transcriptase PCR (RT-PCR) and real- time PCR}

For RT-PCR and real-time PCR quantification analysis of perilipin and SCD1 mRNA expression, total RNA was isolated from 3T3-L1 cells using RiboEX (Geneall), and cDNA was synthesized using MMLV-RTase and oligo (dT) primer (Promega). RT-PCR was performed in 3 individual samples per group and relative mRNA levels of each gene were expressed as the mean $\pm \mathrm{SD}$ of three individual experiments.

Real-time PCR was performed using a Corbett RotorGene 6000 (Qiagen) and relative quantification of PCR product was based on value differences between the target and 36B4 control using the delta-delta CT comparative method. Each group was analyzed in triplicate using total RNA pooled from 3 individual cells. Primer sequences for genes were: perilipin sense; 5'-CTCTGGGAAGCATCGAGAA-3' and antisense; 5'-GATCCACATGGCCAGAGAGT-3', SCD1 sense; 5'-CCTACGACAAGAACATTCAAT-3' and antisense; 5'-CAGGAACTCAGAAGCCCAAAGC-3', 36B4 sense; 5'-GCGTCCTGGCATTGTCTGT-3' and antisense; 5'-GCCGCAAATGCAGATGG-3'.

\section{Gel shift assay}

Gel shift assay was performed as previously described (Kim, et al., 2003). Briefly, TR4, LXR $\alpha$, and RXR $\alpha$ were in vitro synthesized using a T7-quick coupled TNT reticulocyte lysate system (Promega). The following oligomers were used for gel shift assays: consensus DR1 (cDR1) (5'-GATCTCTCTAGGTCAAAGGTCAATTTC-3') and consensus DR4 (cDR4) (5'-GATCACCTCAGGTCA- 


\section{CAGGAGGTCAGACAA-3').}

\section{Results}

TR4 suppresses transcriptional activity of LXR by inhibition of LXR binding to LXRE

Since TR4 and LXR have the binding affinity for DR4 response elements, we first determined whether TR4 could modulate transcriptional activity of $\mathrm{LXR} \alpha$ using reporter genes containing 3 copies of LXR response element (LXRE-Luc). As shown in Fig. 1, transfection of $\mathrm{LXR} \alpha$ and RXR $\alpha$ into NIH-3T3 cells resulted in induction of LXRE-Luc in the presence of $1 \mu \mathrm{M} \mathrm{GW} 3965$. In contrast, TR4 did not induce activity of LXRE-Luc. However, co-transfection of TR4 with LXR $\alpha / \mathrm{RXR} \alpha$ resulted in the suppression of $\mathrm{LXR} \alpha / \mathrm{GW} 3965$-induced luciferase activity. Consistently, we obtained the similar results when HEK293T cells were used for the same reporter gene assay, suggesting that TR4 negatively regulates LXR $\alpha$ transcriptional activity in a cell type-independent manner. Furthermore, the LXR $\alpha$-induced LXRE-Luc activity in NIH-3T3 cells was inhibited by TR4 in a dose-dependent manner.

To dissect the mechanism of how TR4 can repress LXR $\alpha$ transactivation, we used a mammalian two-hybrid assay. Consistent with the previous report (Willy et al., 1995), significant induction was observed when VP16RXR $\alpha$ was co-transfected with GAL4-LXR $\alpha$ (Fig. 2A, lane 6). However, co-transfection of VP16-TR4 with GAL4-LXR $\alpha$ showed near background levels, indicating that TR4 does not form a heterodimer with LXR $\alpha$ (Fig. $2 \mathrm{~A}$, lanes 5). It is also possible that TR4 and LXR $\alpha$ may compete with each other for binding to LXR response element. To determine this possibility, a gel shift assay was performed using $\left[{ }^{32} \mathrm{P}\right]$-labeled LXRE (cDR4). When $\mathrm{LXR} \alpha$ and $\mathrm{RXR} \alpha$ were incubated with $\left[{ }^{32} \mathrm{P}\right]$-labeled LXRE, LXRE formed a complex with LXR $\alpha /$ RXR $\alpha$ (Fig. 2B). In addition, TR4 could also form a specific complex with $\left[{ }^{32} \mathrm{P}\right]$-labeled LXRE. However, when TR4 was added together with $\mathrm{LXR} \alpha / \mathrm{RXR} \alpha, \mathrm{LXR} \alpha / \mathrm{RXR} \alpha$-LXRE complex was reduced in a dose-dependent manner. The data suggest that TR4 inhibition of LXR $\alpha$ transcriptional activity in the reporter gene assays may be due to interruption of the LXR $\alpha / \mathrm{RXR} \alpha$ binding to the LXRE.

\section{Influence of LXR on TR4 transactivation}

LXR $\alpha$ affects transactivation of other nuclear receptors through binding competition for the DNA binding sites (Hashimoto et al., 2009). Thus, we determined the potential regulatory effects of the LXR $\alpha$ on TR4 transactivation using reporter gene assay. As shown in Fig. 3A, TR4 highly induced transcriptional activity of the reporter gene containing 3 copies of TR4RE (TR4RE-Luc) in HEK293T cells, whereas LXR $\alpha$ did not have any effect on this reporter gene. In addition, when LXR $\alpha$ was cotransfected with TR4 into HEK293T and NIH-3T3 cells, TR4-mediated transactivation was not affected by LXR $\alpha$. We also obtained the similar result when we performed the reporter gene assay using cDR1-Luc. To further determine LXR $\alpha$ effect on TR4 binding to TR4RE, we performed a gel shift assay using $\left[{ }^{32} \mathrm{P}\right]$-labeled-TR4RE (cDR1). As shown in Fig. 3B, in vitro translated TR4 could strongly bind to $\left[{ }^{32} \mathrm{P}\right]$-labeled-TR4RE (lane 2, open arrowhead). However, we could not observe any retarded complex when $\mathrm{LXR} \alpha$ and RXR $\alpha$ were incubated with $\left[{ }^{32} \mathrm{P}\right]$-labeledTR4RE. Furthermore, the binding affinity of TR4 for TR4RE was not changed by addition of $L X R \alpha / R X R \alpha$. This data indicates that LXR $\alpha$ could not affect TR4 tran-
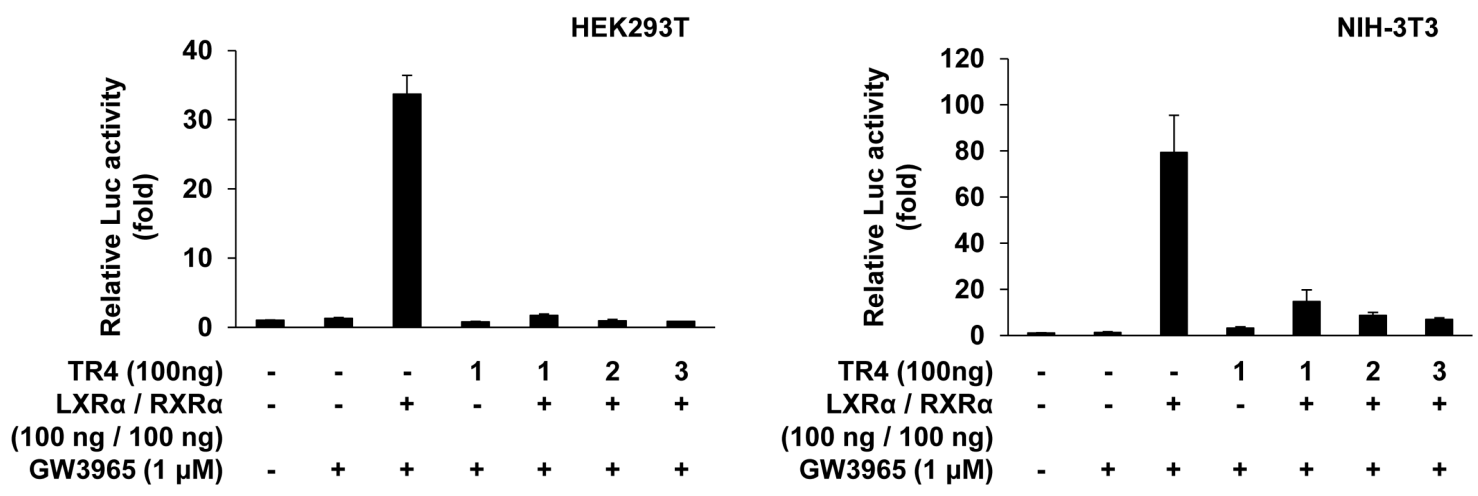

Fig. 1. TR4 inhibits LXR $\alpha$ transcriptional activity. The reporter gene (LXRE-Luc, $300 \mathrm{ng}$ ) was co-transfected with expression plasmids for TR4, LXR $\alpha$, and RXR $\alpha$ into NIH-3T3 and HEK293T cells as indicated. Transfected cells were incubated for $24 \mathrm{~h}$ in the absence or presence of $1 \mu \mathrm{M}$ GW3965 and then harvested to measure luciferase activity. 

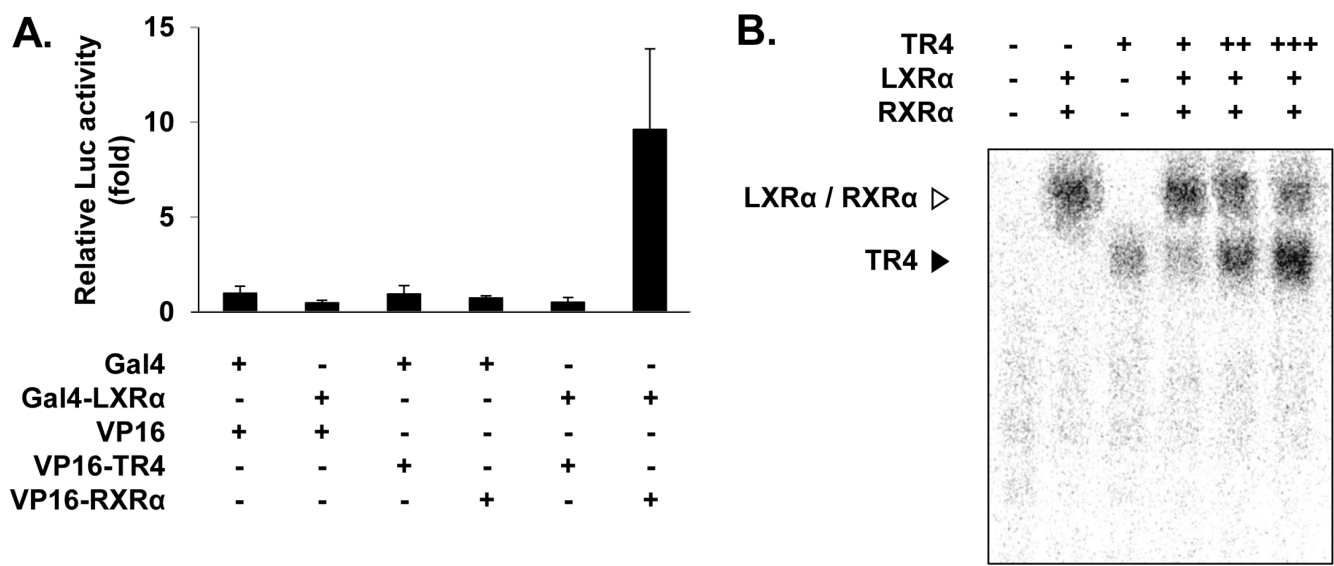

Fig. 2. TR4 competes with $L X R \alpha$ for biding to the $L X R$ responsive element. (A) TR4 does not form a heterodimer with $L X R \alpha$. The Mammalian two-hybrid assay was performed in HEK293T cells by co-transfection of the pG5-Luc (300 ng) with Gal4-LXR $\alpha$, VP16-TR4, and VP16-RXR $\alpha$ (100 ng of each) as indicated and luciferase activity was analyzed. (B) TR4 inhibits LXR $\alpha$ binding to the LXRE. A gel shift assay was performed using $\left.{ }^{32} \mathrm{P}\right]$-labeled LXRE (cDR4) with in vitro translated LXR $\alpha / \mathrm{RXR} \alpha$ and increasing amounts of TR4 as indicated. The retarded LXR $\alpha /$ RXR $\alpha$-LXRE and TR4-LXRE complexes are indicated by open and closed arrowheads, respectively.

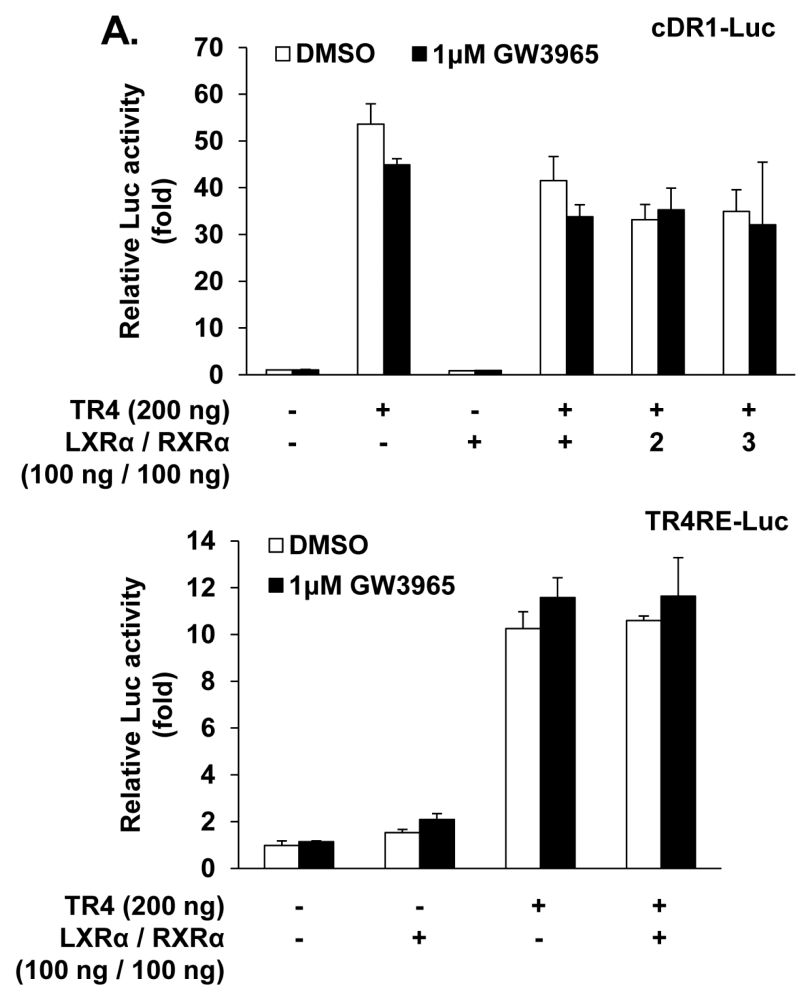

B.

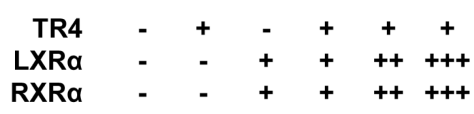

TR4 $\triangleright$

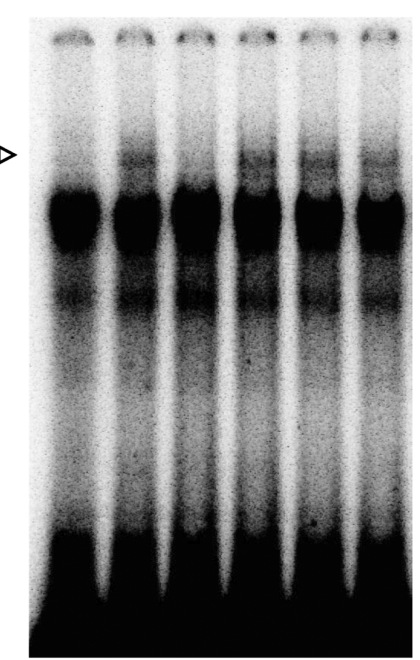

Fig. 3. LXR $\alpha$ does not affect TR4 transcriptional activity and TR4 binding to the TR4RE. (A) Effect of LXR $\alpha$ on TR4 transactivation in HEK293T cells. The indicated reporter genes (300 ng of each) were co-transfected with TR4 expression plasmid and increasing amounts of LXR $\alpha$ and RXR $\alpha$ expression plasmids into HEK293T cells. Transfected cells were incubated for $24 \mathrm{~h}$ in the absence or presence of $1 \mu \mathrm{M}$ GW3965 and then harvested to measure luciferase activity. (B) LXR $\alpha$ is not able to inhibit TR4 binding to the TR4RE. A gel shift assay was performed using $\left[{ }^{32} \mathrm{P}\right]$-labeled TR4RE (cDR1) with in vitro translated TR4 and increasing amounts of $L X R \alpha / R X R \alpha$ as indicated. The retarded TR4-TR4RE complex is indicated by open arrowhead.

scriptional activity due to its inability to inhibit TR4 binding to TR4RE.
TR4 blocks LXR-mediated reduction of lipid accumulation in 3T3-L1 adipocytes

Perilipin is a lipid-droplet associated protein which promotes triacylglycerol storage in adipocytes (Brasaemle et 
al., 2000). LXR is known to downregulate perilipin gene expression in adipocytes (Stenson et al., 2011). Since we also found that TR4 induced perilipin expression, we then determined whether TR4 could modulate the effect of the LXR ligand GW3965 on the expression of perilipin and SCD1 genes in 3T3-L1 adipocytes. We induced differentiation of 2-day post-confluent 3T3-L1 preadipocytes (designated day 0 ) by standard protocol. When $1 \mu \mathrm{M}$ GW3965 was added together with adipogenic stimuli to day 0 3T3-L1 cells and continuously treated throughout the course of differentiation, RT-PCR analysis showed that GW3965 reduced perilipin expression but increased SCD1 expression in day 9 3T3-L1 adipocytes compared with control 3T3-L1 adipocytes (Fig. 4A). In contrast, overexpression of TR4 increased both perilipin and SCD1 expression compared with control 3T3-L1 adipocytes. Conversely, knockdown of TR4 by TR4 microRNA reduced perilipin and SCD1 expression in 3T3-L1 adipocytes. When TR4 was overexpressed in GW3965-treated 3T3-L1 adipocytes, TR4 inhibited GW3965-mediated suppression of perilipin expression. In contrast, co-addition of TR4 and GW3965 resulted in decrease of mRNA level of SCD1 compared with treatment of GW3965 or TR4 overexpression. Next, we determined whether TR4 could affect GW3965-induced decrease of lipid accumu- lation in 3T3-L1 adipocytes using Oil Red O staining. As shown in Fig. 4B, treatment of GW3965 reduced cellular lipid content about $27 \%$ in day 103 T3-L1 adipocytes compared with 3T3-L1 adipocytes treated with vehicle. However, GW3965-mediated reduction of lipid accumulation was completely abolished by TR4 overexpression.

\section{Discussion}

Here we demonstrated an inhibitory role of TR4 in LXR-mediated decrease of lipid accumulation in 3T3-L1 adipocytes by suppressing LXR inhibition of perilipin expression. Furthermore, TR4 negatively regulated LXRa transcriptional activity via binding competition with LXRa for the LXR responsive element.

Nuclear receptors are ligand-activated transcription factors and members of this nuclear receptor family have been considered as key regulators of energy homeostasis in tissues important for energy metabolism (Liu et al., 2007; Powell et al., 2007). A common characteristic of various nuclear receptors is that they play roles as functional regulators of other nuclear receptors since several nuclear receptors use a common response element for the regulation of target gene expression (Lee et al., 1997; Lee et al., 1999). These suggest that mutual regulation
A.

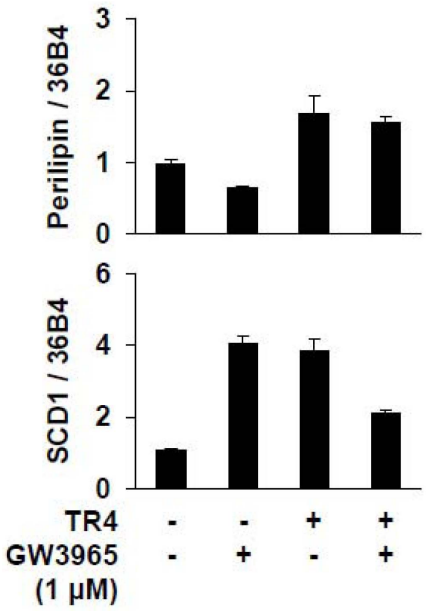

B.
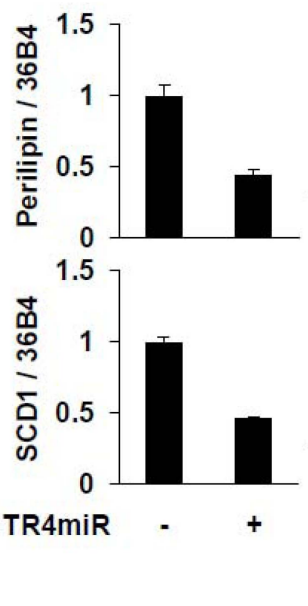

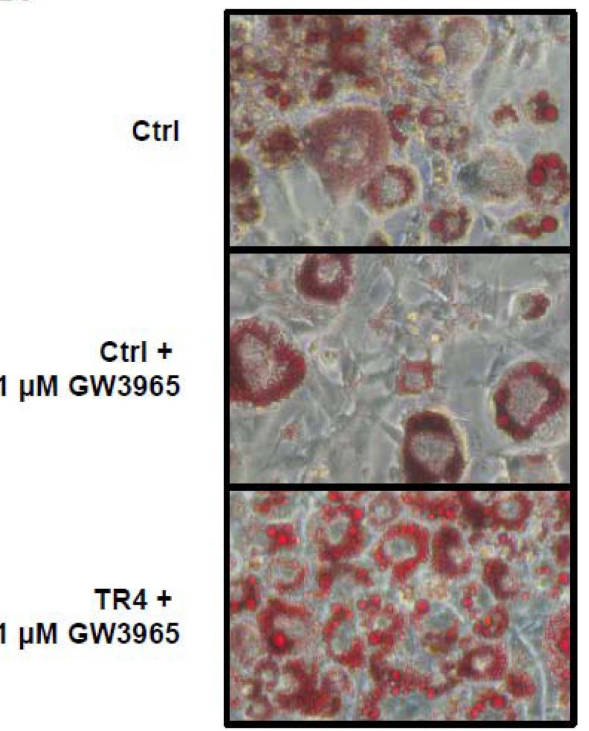

Fig. 4. TR4 inhibits GW3965-induced decrease of perilipin expression and lipid accumulation in 3T3-L1 adipocytes. (A) Effect of TR4 on GW3965-mediated reduction of lipid accumulation in 3T3-L1 adipocytes. At 2 d post-confluence, 3T3-L1 cells stably transfected with empty plasmid, TR4 expression plasmid or TR4 microRNA (TR4miR) were differentiated in the absence or presence of $1 \mu \mathrm{M}$ GW3965 as described in Materials and Methods. Total RNAs prepared from day 9 adipocytes were subjected to RTPCR to determine perilipin and SCD1 mRNA levels. 36B4 fragments were amplified as a loading control. (B) TR4 blocks GW3965- mediated decrease of lipid accumulation in 3T3-L1 adipocytes. Control and TR4 overexpressing 3T3-L1 cells were differentiated in the absence or presence of $1 \mu \mathrm{M} \mathrm{GW} 3965$ for $10 \mathrm{~d}$ as indicated and then lipid accumulation was determined by Oil Red O staining. Oil Red O staining data shown here are representative of three independent experiments with similar results. 
between nuclear receptors in the intracellular environment of adipocytes may lead to control adipocyte development and lipid homeostasis. This prompted us to study the functional cross-talk between TR4 and LXR $\alpha$ in 3T3L1 adipocytes. Previous reports showed that TR4 could induce transcriptional activity of rat $\alpha$-myosin heavychain and S14 genes via DR4 element which are known as a T3RE (Lee et al., 1997). Thus, it is highly possible that TR4 itself may induce transcriptional activity of the LXRE-Luc. However, opposite to our initial assumption, TR4 alone did not affect the activity of the LXRE-Luc. Instead, we observed an inhibitory effect of TR4 on LXRa-induced LXRE-Luc activity. In a gel shift assay, TR4 was able to bind to LXRE and also suppress recruitment of LXRa/RXRa to this LXRE, suggesting that a competition between TR4 and LXRa/RXRa for this DNA element may lead to TR4 inhibition of LXRa transcriptional activity.

Many nuclear receptors can cross-talk with signaling pathways of other nuclear receptors via heterodimerization (Apfel et al., 1994; Shyr et al., 2002). Thus, it is possible that TR4 inhibition of LXRa activity could be occurred via heterodimerization with LXRa. However, TR4 showed no interaction with LXRa in this study. In contrast to negative effect of TR4 on LXRa induction of LXRE-Luc, LXRa did not affect TR4 transcriptional activity. In addition, a gel shift assay revealed that LXR $\alpha$ could not bind to cDR1, suggesting that no effect of LXRa on TR4 transactivation may be due to the lack of its affinity for DR1 element.

Positive effects of LXR $\alpha$ on lipid accumulation during adipocyte differentiation have been reported (Seo et al., 2004; Ulven et al., 2005). However, accumulating evidences suggest that LXR $\alpha$ negatively regulates fat accumulation in adipocytes (Ross et al., 2002; Stenson et al., 2009; Stenson et al., 2011). Furthermore, this inhibitory effect of $\mathrm{LXR} \alpha$ on lipid accumulation in adipocytes is partially due to suppressed expression of perilipin gene and increased basal lipolysis. Recently, we found that TR4 could activate expression of perilipin gene in 3T3L1 adipocytes and also facilitate lipid accumulation via increase of fatty acid uptake (submitted). In this study, TR4 also inhibited LXRa transactivation via binding competition with LXRa for LXRE, strongly implicating that TR4 and LXRa might orchestrate to fine-tune adipocyte physiology. Indeed, GW3965 suppression of perilipin expression in 3T3-L1 adipocytes was abolished by TR4. Consistent with TR4 effect on LXRa regulation of perilipin expression, overexpression of TR4 in 3T3-L1 adipocytes treated with GW3965 abolished GW3965induced decrease of cellular lipid content. Although further study is required to define the role of TR4 in LXRamediated basal lipolysis, our data reveal that TR4 negatively regulates LXRa-induced decrease of lipid accumulation in 3T3-L1 adipocytes. In addition, both TR4 and the LXRa/RXRa induced expression of SCD1 in 3T3-L1 adipocytes and these results were consistent with previous reports. Interestingly, when both TR4 and GW3965 were added to 3T3-L1 cells, mRNA level of SCD1 was reduced as compared with those of TR4 overexpressing or GW3965-treated cells, suggesting that metabolic cues may signal TR4 and LXRa to regulate SCD1 dynamically under physiological conditions, leading to multiple transcriptional responses.

In conclusion, we have shown that TR4 plays an inhibitory role in LXR-mediated reduction of lipid accumulation in 3T3-L1 adipocytes via blocking negative effect of LXR on perilipin expression.

\section{Acknowledgement}

This work was supported by National Research Foundation of Korea Grant funded by the Korean Government (KRF-2006-312-C00392).

\section{References}

1. Apfel, R., Benbrook, D., Lernhardt, E., Ortiz, M. A., Salbert, G., and Pfahl, M. (1994) A novel orphan receptor specific for a subset of thyroid hormone-responsive elements and its interaction with the retinoid/thyroid hormone receptor subfamily. Mol. Cell. Biol. 14, 7025-7035.

2. Brasaemle, D. L., Rubin, B., Harten, I.A., Gruia-Gray, J., Kimmel, A. R., and Londos, C. (2000) Perilipin A increases triacylglycerol storage by decreasing the rate of triacylglycerol hydrolysis. J. Biol. Chem. 275, 38486-38493.

3. Hashimoto, K., Ishida, E., Matsumoto, S., Okada, S., Yamada, M., Satoh, T., Monden, T., and Mori, M. (2009) Carbohydrate response element binding protein gene expression is positively regulated by thyroid hormone. Endocrinology 150, 3417-3424.

4. Hunnicutt, J. W., Hardy, R. W., Williford, J., and McDonald, J. M. (1994) Saturated fatty acid-induced insulin resistance in rat adipocytes. Diabetes 43, 540-545.

5. Kim, E., Liu, N. C., Yu, I. C., Lin, H. Y., Lee, Y. F., Sparks, J. D., Chen, L. M., and Chang, C. (2011a) Metformin inhibits nuclear receptor TR4-mediated hepatic stearoyl-coenzyme A desaturase 1 gene expression with altered insulin sensitivity. Diabetes 60, 1493-1503.

6. Kim, E., Xie, S., Yeh, S. D., Lee, Y. F., Collins, L. L., Hu, Y. C., Shyr, C. R., Mu, X. M., Liu, N. C., Chen, Y. T., Wang, P. 
H., and Chang, C. (2003) Disruption of TR4 orphan nuclear receptor reduces the expression of liver apolipoprotein E/CI/C-II gene cluster. J. Biol. Chem. 278, 46919-46926.

7. Kim, S. J., Choi, H., Park, S. S., Chang, C., and Kim, E. (2011b) Stearoyl CoA desaturase (SCD) facilitates proliferation of prostate cancer cells through enhancement of androgen receptor transactivation. Mol. Cells 31, 371-377.

8. Kim, S. J., Choi, H., Jung, C. H., Park, S. S., Cho, S. R., Oh, S., and Kim, E. (2010) Calcium mobilization inhibits lipid accumulation during the late adipogenesis via suppression of PPAR gamma and LXR alpha signalings. Korean J. Food Sci. Ani. Resour. 30, 787-794.

9. Lee, C. H., Chinpaisal, C., and Wei, L. N. (1998a) A novel nuclear receptor heterodimerization pathway mediated by orphan receptors TR2 and TR4. J. Biol. Chem. 273, 2520925215.

10. Lee, H. J., Lee, Y., Burbach, J. P., and Chang, C. (1995) Suppression of gene expression on the simian virus 40 major late promoter by human TR4 orphan receptor. A member of the steroid receptor superfamily. J. Biol. Chem. 270, 3012930133.

11. Lee, Y. F., Young, W. J., Burbach, J. P., and Chang, C. (1998b) Negative feedback control of the retinoid-retinoic acid/retinoid X receptor pathway by the human TR4 orphan receptor, a member of the steroid receptor superfamily. $J$. Biol. Chem. 273, 13437-13443.

12. Lee, Y. F., Pan, H. J., Burbach, J. P., Morkin, E., and Chang, C. (1997) Identification of direct repeat 4 as a positive regulatory element for the human TR4 orphan receptor. A modulator for the thyroid hormone target genes. J. Biol. Chem. 272, 12215-12220.

13. Lee, Y. F., Young, W. J., Lin, W. J., Shyr, C. R., and Chang, C. (1999) Differential regulation of direct repeat 3 vitamin D3 and direct repeat 4 thyroid hormone signaling pathways by the human TR4 orphan receptor. J. Biol. Chem. 274, 16198-16205.

14. Liu, N. C., Lin, W. J., Kim, E., Collins, L. L., Lin, H. Y., Yu, I. C., Sparks, J. D., Chen, L. M., Lee, Y. F., and Chang, C. (2007) Loss of TR4 orphan nuclear receptor reduces phosphoenolpyruvate carboxykinase-mediated gluconeogenesis. Diabetes 56, 2901-2909.

15. Ory, D. S. (2004) Nuclear receptor signaling in the control of cholesterol homeostasis: have the orphans found a home? Circ. Res. 95, 660-670.

16. Powell, E., Kuhn, P., and Xu, W. (2007) Nuclear Receptor Cofactors in PPAR gamma-mediated adipogenesis and adipocyte energy metabolism. PPAR Res. 2007, 53843.
17. Reaven, G. M., Hollenbeck, C., Jeng, C. Y., Wu, M. S., and Chen, Y. D. (1988) Measurement of plasma glucose, free fatty acid, lactate, and insulin for $24 \mathrm{~h}$ in patients with NIDDM. Diabetes 37, 1020-1024.

18. Ross, S. E., Erickson, R. L., Gerin, I., DeRose, P. M., Bajnok, L., Longo, K. A., Misek, D. E., Kuick, R., Hanash, S. M., Atkins, K. B., Andresen, S. M., Nebb, H. I., Madsen, L., Kristiansen, K., and MacDougald, O. A. (2002) Microarray analyses during adipogenesis: understanding the effects of Wnt signaling on adipogenesis and the roles of liver $\mathrm{X}$ receptor alpha in adipocyte metabolism. Mol. Cell Biol. 22, 59895999.

19. Seo, J. B., Moon, H. M., Kim, W. S., Lee, Y. S., Jeong, H. W., Yoo, E. J., Ham, J., Kang, H., Park, M. G., Steffensen, K. R., Stulnig, T. M., Gustafsson, J. A., Park, S. D., and Kim, J. B. (2004) Activated liver X receptors stimulate adipocyte differentiation through induction of peroxisome proliferatoractivated receptor gamma expression. Mol. Cell. Biol. 24, 3430-3444.

20. Shyr, C. R., Hu, Y. C., Kim, E., and Chang, C. (2002) Modulation of estrogen receptor-mediated transactivation by orphan receptor TR4 in MCF-7 cells. J. Biol. Chem. 277, 14622-14628.

21. Stenson, B. M., Ryden, M., Steffensen, K. R., Wahlen, K., Pettersson, A. T., Jocken, J. W., Arner, P., and Laurencikiene, J. (2009) Activation of liver X receptor regulates substrate oxidation in white adipocytes. Endocrinology 150, 41044113.

22. Stenson, B. M., Ryden, M., Venteclef, N., Dahlman, I., Pettersson, A. M., Mairal, A., Astrom, G., Blomqvist, L., Wang, V., Jocken, J. W., Clement, K., Langin, D., Arner, P., and Laurencikiene, J. (2011) Liver X receptor (LXR) regulates human adipocyte lipolysis. J. Biol. Chem. 286, 370-379.

23. Ulven, S. M., Dalen, K. T., Gustafsson, J. A., and Nebb, H. I. (2005) LXR is crucial in lipid metabolism. Prostaglandins Leukot. Essent. Fatty Acids 73, 59-63.

24. Willy, P. J., Umesono, K., Ong, E. S., Evans, R. M., Heyman, R. A., and Mangelsdorf, D. J. (1995) LXR, a nuclear receptor that defines a distinct retinoid response pathway. Genes Dev. 9, 1033-1045.

25. Zidi, A., Fernandez-Cabanas, V. M., Urrutia, B., Carrizosa, J., Polvillo, O., Gonzalez-Redondo, P., Jordana, J., Gallardo, D., Amills, M., and Serradilla, J. M. (2010) Association between the polymorphism of the goat stearoyl-CoA desaturase 1 (SCD1) gene and milk fatty acid composition in Murciano-Granadina goats. J. Dairy Sci. 93, 4332-4339.

(Received 2011.5.4/Revised 2011.6.8/Accepted 2011.6.8) 